\title{
Linear BVPs and SIEs for Generalized Regular Functions in Clifford Analysis
}

\author{
Pingrun Li $\mathbb{D i D}^{1}$ and Lixia Cao ${ }^{2}$ \\ ${ }^{1}$ School of Mathematical Sciences, Qufu Normal University, Qufu 273165, China \\ ${ }^{2}$ Mathematics College, Northeast Petroleum University, Daqing 163318, China \\ Correspondence should be addressed to Pingrun Li; lipingrun@163.com
}

Received 5 December 2017; Revised 6 March 2018; Accepted 14 March 2018; Published 8 May 2018

Academic Editor: Richard I. Avery

Copyright (c) 2018 Pingrun Li and Lixia Cao. This is an open access article distributed under the Creative Commons Attribution License, which permits unrestricted use, distribution, and reproduction in any medium, provided the original work is properly cited.

We study some properties of a regular function in Clifford analysis and generalize Liouville theorem and Plemelj formula with values in Clifford algebra $A_{n}(\mathbb{R})$. By means of the classical Riemann boundary value problem and of the theory of a regular function, we discuss some boundary value problems and singular integral equations in Clifford analysis and obtain the explicit solutions and the conditions of solvability. Thus, the results in this paper will be of great significance for the study of improving and developing complex analysis, integral equation, and boundary value theory.

\section{Introduction}

As we know, boundary value problems (BVPs) for holomorphic function and singular integral equations (SIEs) are one of important branches in classical holomorphic function theory of one complex variable, and they have important applications in many fields, such as mechanics, physics, and engineering. Many practical problems can often be transformed into BVPs and SIEs. In recent decades, some kinds of BVPs and SIEs have been well studied and a lot of results were obtained (see, e.g., [1-6]). Clifford analysis is an important field of modern mathematics which studies the functions defined in $\mathbb{R}^{n}$ with values in Clifford algebra space $A_{n}(\mathbb{R})$ and possesses both theoretical and applicable values, such as physics, quantum mechanics, Maxwell equation, theory of Yang-Mills field, and other branches of mathematics (see, e.g., [7-10]). The results of theory of Clifford regular function are generalizations of the classical theory of holomorphic functions in complex analysis. A lot of results of the classical theory of holomorphic functions can be extended to Clifford analysis. In recent decades, many mathematical workers devote to study the BVPs and SIEs in Clifford analysis, and there were many important and significant results (see, e.g., $[11-13])$.
Based on the above-mentioned work, we further solve some kinds of BVPs and SIEs in Clifford analysis as well as BVP in quaternion analysis. We first study some properties of a regular function and then generalize Cauchy integral theorem and Cauchy integral formula in $A_{n}(\mathbb{R})$. By means of the Riemann boundary value problem and of the properties of the Cauchy principal value integral we obtain the explicit expressions of general solution and their solvability conditions for these equations. Thus, this paper generalizes the theory of integral equations and the classical boundary value problems for analytic function.

We begin by developing the necessary preliminaries in Clifford algebra and analysis that we require here.

\section{Preliminaries}

Let $A_{n}(\mathbb{R})$ be a real Clifford algebra over an $n$-dimensional real vector space $\mathbb{R}^{n}$ with the orthogonal basis $\left\{e_{1}, e_{2}, \ldots, e_{n}\right\}$, where $e_{1}=1$ is an identity element. The product on $\mathbb{R}^{n}$ is defined by

$$
\begin{aligned}
e_{1}^{2} & =1, \\
e_{i}^{2} & =-1 \quad(i=2,3, \ldots, n), \\
e_{1} e_{i} & =e_{i} e_{1} \quad(i=2,3, \ldots, n),
\end{aligned}
$$




$$
e_{i} e_{j}+e_{j} e_{i}=\left\{\begin{array}{ll}
0, & i \neq j ; \\
-2, & i=j
\end{array} \quad(2 \leq i, j \leq n)\right.
$$

Hence, any element $x \in \mathbb{R}^{n}$ has the type $x=\sum_{k=1}^{n} x_{k} e_{k}\left(x_{k} \in\right.$ $\mathbb{R})$. And $A_{n}(\mathbb{R})$ is a $2^{n-1}$-dimensional real linear space, whose basis is $\left\{e_{1}, e_{2}, \ldots, e_{n} ; e_{2} e_{3}, \ldots, e_{n-1} e_{n} ; \ldots ; e_{2} e_{3}, \ldots, e_{n}\right\}$. Each basis element of $A_{n}(\mathbb{R})$ has a representation of the form $e_{A}=e_{\alpha_{1} \alpha_{2} \cdots \alpha_{r}}=e_{\alpha_{1}} e_{\alpha_{2}} \cdots e_{\alpha_{r}}$, where $A=\left\{\alpha_{1}, \alpha_{2}, \ldots, \alpha_{r}\right\} \subseteq$ $\{1,2, \ldots, n\}$ and $1 \leq \alpha_{1}<\alpha_{2}<\cdots<\alpha_{r} \leq n$, and when $A=1, \alpha_{1}=e_{1}=1$. Therefore, any element $a \in A_{n}(\mathbb{R})$ can be denoted as $a=\sum_{A} a_{A} e_{A}$, where $a_{A} \in \mathbb{R}$ is a real number. The norm for an element $a \in A_{n}(\mathbb{R})$ is taken to be

$$
|a|=\sqrt{(a, a)}=\sqrt{|a|^{2}}=\sqrt{\sum_{A}\left|a_{A}\right|^{2}} .
$$

It is easy to prove that, for any $a, b \in A_{n}(\mathbb{R})$, we have

$$
\begin{aligned}
|a+b| & \leq|a|+|b|, \\
|a b| & \leq 2^{n-1}|a||b| .
\end{aligned}
$$

In fact, we denote $a=\sum_{A} a_{A} e_{A}, b=\sum_{A} b_{A} e_{A}$, then

$$
\begin{aligned}
|a+b| & \leq \sqrt{\sum_{A}\left(\left|a_{A}\right|+\left|b_{A}\right|\right)^{2}} \\
& \leq\left(\sum_{A}\left|a_{A}\right|^{2}\right)^{1 / 2}+\left(\sum_{A}\left|b_{A}\right|^{2}\right)^{1 / 2}=|a|+|b| \\
|a b| & \leq \sqrt{\sum_{A} 2^{2 n}\left|a_{A}\right|^{2}\left|b_{A}\right|^{2}} \\
& \leq 2^{n-1}\left(\sum_{A}\left|a_{A}\right|^{2}\right)^{1 / 2}\left(\sum_{A}\left|b_{A}\right|^{2}\right)^{1 / 2} \\
& =2^{n-1}|a||b| .
\end{aligned}
$$

Definition 1. Let $\Omega \subset \mathbb{R}^{n}$ be a nonempty, open, and connected set. A function $f(x)$ defined in $\Omega$ and with values in Clifford algebra $A_{n}(\mathbb{R})$ can be expressed as $f(x)=\sum_{A} f_{A}(x) e_{A}$; that is, $f: \Omega \rightarrow A_{n}(\mathbb{R})$, where $f_{A}(x)$ is a real function.

Let $C^{(r)}(\Omega)$ denote the set of all functions defined in $\Omega$ with derivatives of order $r(\geq 1)$. We define the function class $F_{\Omega}^{(r)}$ in $C^{(r)}(\Omega)$ as follows:

$$
\begin{aligned}
F_{\Omega}^{(r)} & =\left\{f \mid f: \Omega \longrightarrow A_{n}(\mathbb{R}), f(x)\right. \\
& \left.=\sum_{A} f_{A}(x) e_{A}, f_{A}(x) \in C^{(r)}(\Omega), x \in \Omega\right\},
\end{aligned}
$$

where $\Omega \subset \mathbb{R}^{n}$ and $r(\geq 1)$ is an integer.
Definition 2. Let $f: \Omega \rightarrow A_{n}(\mathbb{R})$. If there exists a constant $M>0$ such that

$$
\left|f\left(x_{1}\right)-f\left(x_{2}\right)\right| \leq M\left|x_{1}-x_{2}\right|^{\alpha}, \quad 0<\alpha \leq 1,
$$

for any $x_{1}, x_{2} \in \Omega$, then we call $f$ to be Hölder continuous function in $\Omega$, denoted by $f \in H_{\Omega}^{\alpha}$, where $M$ and $\alpha$ are called the Hölder constant and the Hölder index, respectively. And we define generalized Cauchy-Riemann operators (i.e., Dirac operator) $D, \bar{D}$ in $F_{\Omega}^{(r)}$ as follows:

$$
\begin{aligned}
& D=e_{1} \partial_{1}-\sum_{i=2}^{n} e_{i} \partial_{i}, \\
& \bar{D}=\sum_{i=1}^{n} e_{i} \partial_{i} .
\end{aligned}
$$

For any $f \in F_{\Omega}^{(r)}(r \geq 1)$, the operators $D, \bar{D}$ act on function $f$ being governed by the rules

$$
\begin{aligned}
& D_{x} f=\sum_{i=1}^{n} \sum_{A} e_{i} e_{A} \frac{\partial f_{A}}{\partial x_{i}}, \\
& f D_{x}=\sum_{A} \sum_{i=1}^{n} e_{A} e_{i} \frac{\partial f_{A}}{\partial x_{i}},
\end{aligned}
$$

then a solution of $D_{x} f=0$ is called left regular function in $\Omega$. Similarly, a solution of $f D_{x}=0$ is called right regular function. Generally the left regular function is called regular function in short.

Let the boundary $\partial \Omega$ of $\Omega$ be a smooth, directional, and compact Liapunov surface. We denote the set of all Hölder continuous function defined on $\partial \Omega$ as $H(\partial \Omega, \beta)$ and define the norm as

$$
\|f\|_{\beta}=C(f, \partial \Omega)+H(f, \partial \Omega, \beta),
$$

where $C(f, \partial \Omega)=\max _{t \in \partial \Omega}|f(t)|$ is a continuous norm, and

$$
H(f, \partial \Omega, \beta)=\sup _{t_{1} \neq t_{2}, t_{1}, t_{2} \in \partial \Omega} \frac{\left|f\left(t_{1}\right)-f\left(t_{2}\right)\right|}{\left|t_{1}-t_{2}\right|^{\beta}}
$$

is an Hölder norm. It is easy to prove that $H(\partial \Omega, \beta)$ is a Banach space.

Definition 3. If there exists $b\left(b \in A_{n}(\mathbb{R})\right)$ such that $a b=b a=$ 1 , we call $b$ as the inverse of $a$, denoted by $a^{-1}$.

In the following, $x \in \mathbb{R}^{n}$ can be expressed as $x=\sum_{i=1}^{n} x_{i} e_{i}$, $x=x_{R}+x_{n} e_{n}$, or $x=\left(x_{R}, x_{n}\right)$, where $x_{R}=\sum_{i=1}^{n-1} e_{i} x_{i}$. Thus, $\mathbb{R}^{n}$ can be divided into three parts: $\mathbb{R}_{+}^{n}=\left\{x \mid x_{n}>0\right\}, \mathbb{R}_{-}^{n}=$ $\left\{x \mid x_{n}<0\right\}$, and $\mathbb{R}_{0}^{n}=\left\{x \mid x_{n}=0\right\}$. Obviously, $\overline{\mathbb{R}}_{+}^{n}=\mathbb{R}_{+}^{n} \cup \mathbb{R}_{0}^{n}$ and $\overline{\mathbb{R}}_{-}^{n}=\mathbb{R}_{-}^{n} \cup \mathbb{R}_{0}^{n}$. However, $\mathbb{R}_{0}^{n}$ can also be represented by $\mathbb{R}^{n-1}$, and $\mathbb{R}_{+}^{n}, \mathbb{R}_{-}^{n}$ lie in the upper and lower half spaces of the hyperplane $\mathbb{R}_{0}^{n}$, respectively.

The conjugate of a number $a$ is the number $\bar{a}$ given by $\bar{a}=$ $\sum_{A} a_{A} \bar{e}_{A}$, where $\bar{e}_{A}=\bar{e}_{\alpha_{1}} \bar{e}_{\alpha_{2}} \ldots \bar{e}_{\alpha_{j}}, \bar{e}_{j}=-e_{j}(j=1,2, \ldots n)$. 
For any $x \in \mathbb{R}^{n}$, we know that $x \bar{x}=-|x|^{2}$, and we can verify that $x^{-1}=-\bar{x} /|x|^{2}$ is an inverse element of $x \neq 0$; that is, $x x^{-1}=x^{-1} x=1$.

Let $A_{n-1}(\mathbb{R})$ be the subalgebra constructed by $\left\{e_{1}, e_{2}, \ldots\right.$, $\left.e_{n-1}\right\}$, then $A_{n-1}(\mathbb{R}) \subseteq A_{n}(\mathbb{R})$ and $A_{n}(\mathbb{R})$ has the decomposition $A_{n}(\mathbb{R})=A_{n-1}(\mathbb{R})+e_{n} A_{n-1}(\mathbb{R})$. It is clear that the decomposition is the generalization of the classical representation of a complex number. Thus, any $x=\sum_{A} x_{A} e_{A} \in \mathbb{R}_{n}(A)$ can be decomposed as $x=b+c e_{n}$, where $b, c \in A_{n-1}(\mathbb{R})$. We may define operators $P, Q$ as $P x=b, Q x=c$.

Definition 4. Let $f(x)$ be defined in $\mathbb{R}^{n}, a \in \mathbb{R}^{n}$. If $D_{x} f(a)=$ 0 , then we call $a$ to be a regular point. If $D_{x} f(a) \neq 0$, but there always exists $b$ in any neighborhood of $a$ such that $D_{x} f(b)=$ 0 , then we call $a$ to be a singular point. If there is a hollow neighborhood $U(a)$ of $a$ such that $D_{x} f(x)=0$ for any $x \in$ $U(\bar{a})=U(a)-\{a\}$, we call $a$ to be an isolated point of $f(x)$.

Definition 5. If $f(x)$ is a regular function in $\mathbb{R}^{n}$, that is, $D f(x)=0$ and $f(x) \in F_{\Omega}^{(r)}$, then we say that $f(x)$ is an entire function in $\mathbb{R}^{n}$, and $x=\infty$ is the only singular point of $f(x)$ in $\mathbb{R}^{n}$.

\section{Some Lemmas and Their Properties}

In this section we present some lemmas, which are important to our results.

Lemma 6 ((Plemelj formula) (see [7])). Let

$$
F(x)=\frac{1}{w_{n-1}} \int_{\partial \Omega} \frac{\overline{t-x}}{|t-x|^{n}} \theta(t) f(t) d S t,
$$

$x \in \mathbb{R}^{n} \backslash \partial \Omega$,

where $w_{n-1}=2 \pi^{n / 2} / \Gamma(n / 2)$ denotes the surface area of a unit sphere in $\mathbb{R}^{n}$ and $\theta(t)=\sum_{j=1}^{n} e_{j} \cos \left(m, e_{j}\right) . m$ is a normal vector at $t \in \partial \Omega$, and $d S t$ is a Lebesgue measure on $\partial \Omega$. $f(t)$ is a bounded Hölder continuous function on $\partial \Omega$, that is, $f(t) \in H(\partial \Omega, \beta)(0<\beta \leq 1)$, then

$$
F^{ \pm}(x)= \pm \frac{1}{2} f(x)+(P f)(x), \quad x \in \partial \Omega,
$$

where

$$
(P f)(x)=\frac{1}{w_{n-1}} \int_{\partial \Omega} \frac{\overline{t-x}}{|t-x|^{n}} \theta(t) f(t) d S t, \quad x \in \partial \Omega .
$$

Lemma 7. Let $t, x \in \mathbb{R}^{n}(n \geq 2)$ and $m \geq 0$ be an integer. Then one has

$$
\begin{aligned}
& \left|\frac{\overline{t-x_{1}}}{\left|t-x_{1}\right|^{n}}-\frac{\overline{t-x_{2}}}{\left|t-x_{2}\right|^{n}}\right| \\
& \quad \leq \frac{B_{m}\left(x_{1}, x_{2}, t\right)}{\left|t-x_{1}\right|^{n-1}\left|t-x_{2}\right|^{n-2}} \cdot\left|x_{1}-x_{2}\right|,
\end{aligned}
$$

where

$$
B_{m}\left(x_{1}, x_{2}, t\right)= \begin{cases}\sum_{t=0}^{m}\left|t-x_{1}\right|^{m-k}\left|t-x_{2}\right|^{k}, & m>0 ; \\ 1, & m=0 .\end{cases}
$$

Proof. It can easily be proved by a direct calculation.

Lemma 8. If $f(x) \in H(\partial \Omega, \beta)$, then $(P f)(x) \in H(\partial \Omega, \beta)$ and $F^{ \pm}(x) \in H(\partial \Omega, \beta)$.

Proof. In order to prove that $(P f)(x) \in H(\partial \Omega, \beta)$, we consider

$$
\begin{aligned}
& \left|(P f)\left(x_{1}\right)-(P f)\left(x_{2}\right)\right|=\mid \frac{1}{w_{n-1}} \\
& \cdot \int_{\partial \Omega} \frac{\overline{t-x_{1}}}{\left|t-x_{1}\right|^{n}} \theta(t) f(t) d S t-\frac{1}{w_{n-1}} \\
& \cdot \int_{\partial \Omega} \frac{\overline{t-x_{2}}}{\left|t-x_{2}\right|^{n}} \theta(t) f(t) d S t|=| \frac{1}{w_{n-1}} \\
& \cdot \int_{\partial \Omega}\left[\frac{\overline{t-x_{1}}}{\left|t-x_{1}\right|^{n}}-\frac{\overline{t-x_{2}}}{\left|t-x_{2}\right|^{n}}\right] \theta(t) f(t) d S t \mid \\
& \leq\left|\frac{1}{w_{n-1}} \int_{\partial \Omega} \frac{B_{m}}{\left.\mid t-x_{1}, x_{2}, t\right) f(t) \theta(t)} d S t\right| \cdot \mid x_{1} \\
& -\left.x_{2}\right|^{\beta-2}=M\left|t-x_{2}\right|^{n-2}-\left.x_{2}\right|^{\beta},
\end{aligned}
$$

where

$$
M=\left|\frac{1}{w_{n-1}} \int_{\partial \Omega} \frac{B_{m}\left(x_{1}, x_{2}, t\right) f(t) \theta(t)}{\left|t-x_{1}\right|^{n-2}\left|t-x_{2}\right|^{n-2}} d S t\right|
$$

that is, there exists a constant $M>0$ such that $\mid(P f)\left(x_{1}\right)-$ $(P f)\left(x_{2}\right)|\leq M| x_{1}-\left.x_{2}\right|^{\beta}$. Therefore, we get $(P f)(x) \in$ $H(\partial \Omega, \beta)$.

Similarly, by Hile lemma (see [12]) we can prove $\| \pm f / 2+$ $P f\left\|_{\beta} \leq C\right\| f \|_{\beta}$, thus, $F^{ \pm}(x) \in H(\partial \Omega, \beta)$.

The proof of Lemma 8 is complete.

Lemma 9 (see [13]). Let $\Omega$ be a bounded domain in $\mathbb{R}^{n}$, then the integral

$$
P . V . \frac{1}{w_{n-1}} \int_{\partial \Omega} \frac{\overline{t-x}}{|t-x|^{n}} \theta(t) d S t, \quad x \in \partial \Omega ;
$$

that is,

$$
\frac{1}{w_{n-1}} \int_{\partial \Omega} \frac{\overline{t-x}}{|t-x|^{n}} \theta(t) d S t
$$

exists under the meaning of Cauchy principal value, and its value is equal to $1 / 2$, where $\partial \Omega$ and $\theta(t)$ are the same as before.

Lemma 10 (see [14]). Let $f(x) \in H(\partial \Omega, \beta)$, then the Cauchy type integral

$$
F(x)=\frac{1}{w_{n-1}} \int_{\partial \Omega} \frac{\overline{t-x}}{|t-x|^{n}} \theta(t) f(t) d S t, \quad x \notin \partial \Omega
$$

is a regular function in $\Omega$, and $D_{x} F(x)=0, F(\infty)=0$.

The following Lemmas 11-13 are obvious facts, and their proofs are similar to that of the classical theorems. More details will be omitted here. 
Lemma 11 (generalized Liouville theorem). Let $f(x)$ be a regular function in $\mathbb{R}^{n}$ and $f(x) \in F_{\Omega}^{(r)}(r \geq 1)$; that is, $f(x)$ is an entire function. Then one has the following:

(1) If $f(\infty)=0$, then $f(x) \equiv 0$.

(2) If $f(\infty)=C$, then $f(x) \equiv C$, where $C$ is a Clifford constant.

(3) If $|f(x)| \leq M$ (constant) for any $x \in \mathbb{R}^{n}$, then $f(x)$ is a constant in $\mathbb{R}^{n}$.

(4) If $\lim _{x \rightarrow \infty} f(x)$ exists, then $f(x)$ is a constant in $\mathbb{R}^{n}$.

Lemma 12 (generalized Cauchy integral theorem). Let $f(x)$ be a regular function in $\Omega \subset \mathbb{R}^{n}$ and $\widetilde{\Omega}$ be a single connected domain of $\Omega$. For any smooth, closed, and rectifiable curve, $\gamma \in$ $\widetilde{\Omega}$. Then

$$
\frac{1}{w_{n-1}} \int_{\gamma} \frac{\overline{t-x}}{|t-x|^{n}} \theta(t) f(t) d S t=0, \quad x \in \widetilde{\Omega} .
$$

Lemma 13 (generalized Cauchy integral formula). Let $f(x)$ be a regular function in $\Omega \subset \mathbb{R}^{n}$ and $U \subset \Omega$ be a bounded domain. $\partial U$ is a smooth, differentiable, and compact surfaces. Then

$$
f(z)=\frac{1}{w_{n-1}} \int_{\partial U} \frac{\overline{t-z}}{|t-z|^{n}} \theta(t) f(t) d S t
$$

for any $z \in U$, where $U \cup \partial U=\bar{U} \subset \Omega$.

\section{BVP in Clifford Analysis}

Let $\Omega \subset \mathbb{R}^{n}$ be an open and connected set and the boundary $\partial \Omega$ of $\Omega$ be a smooth, compact, oriented, and closed Liapunov surface. $\mathbb{R}^{n}$ is divided into two domains $\Omega^{+}=\Omega$ and $\Omega^{-}=$ $\mathbb{R}^{n} \backslash \Omega$ by $\partial \Omega$, with $\infty \in \Omega^{-}$. Our goal is to obtain an $A_{n}(\mathbb{R})$-valued function $f(t)$ such that it is regular in $\Omega^{ \pm}$and continuous on $\Omega^{ \pm}+\partial \Omega$, and we have the following boundary value condition:

$$
f^{+}(t)=f^{-}(t) B+h(t), \quad t \in \partial \Omega,
$$

where $B \in A_{n}(\mathbb{R})$ is a given Clifford constant with $B \neq 0$ and $B$ right inverse $B^{-1}$. A known function $h(t) \in H(\partial \Omega, \beta) . f^{ \pm}(t)$ are the boundary values of $f(t)$ on $\partial \Omega$, respectively. If the order of $f(t)$ is $m$ at infinity, such a problem can be denoted as $R_{m}$. Actually, the problem $R_{0}$ and problem $R_{-1}$ are frequently discussed. On the problem $R_{0}, f(\infty)$ is supposed to be finite and nonzero. On $R_{-1}, f(\infty)$ is assumed to be zero. In order that a solution of (23) exists, we require $f(t) \in H(\partial \Omega, \beta)$ (i.e., $\left.f^{ \pm}(t) \in H(\partial \Omega, \beta)\right)$ and the following condition is fulfilled:

$$
\frac{1}{w_{n-1}} \int_{\partial \Omega} \frac{|f(t)|}{\left(1+|t|^{2}\right)^{(1 / 2)(n-1)}} d S t<+\infty,
$$

where $d S t$ is a unit area element on $\partial \Omega$. Consider

$$
F(x)=\frac{1}{w_{n-1}} \int_{\partial \Omega} \frac{\overline{t-x}}{|t-x|^{n}} \theta(t) f(t) d S t,
$$

where $w_{n-1}$ is the surface area of a unit sphere in $\mathbb{R}^{n}$ and $\theta(t)=$ $\sum_{j=1}^{n} e_{j} \cos \left(m, e_{j}\right) ; m$ is an outer normal vector at $t \in \partial \Omega$. By Lemmas 6-8 we have

$$
F^{ \pm}(x)= \pm \frac{1}{2} f(x)+(P f)(x), \quad x \in \partial \Omega,
$$

where

$$
(P f)(x)=\frac{1}{w_{n-1}} \int_{\partial \Omega} \frac{\overline{t-x}}{|t-x|^{n}} \theta(t) f(t) d S t,
$$

$x \in \partial \Omega$.

Under condition (24), $F(x)$ is regular for all $x \in \mathbb{R}^{n} \backslash \Omega$ and $D_{x} F(x)=0$. And $(P f)(x)$ is a singular integral and it is convergent under the meaning of Cauchy principal value. Moreover, $F(x)$ is continuously extended to the boundary $\partial \Omega$ form $\Omega$ and $\mathbb{R}^{n} \backslash \Omega$, respectively, and then obtain the boundary values $F^{+}(x)$ and $F^{-}(x)$. Note that

$$
\begin{aligned}
(P f)(x) & =\frac{1}{w_{n-1}} \int_{\partial \Omega} \frac{\overline{t-x}}{|t-x|^{n}} \theta(t) f(t) d S t \\
& =\lim _{\delta \rightarrow 0^{+}} \frac{1}{w_{n-1}} \int_{|t-x| \geq \delta} \frac{\overline{t-x}}{|t-x|^{n}} \theta(t) f(t) d S t, \\
& x \in \partial \Omega .
\end{aligned}
$$

Particularly, when $f(t)=1$, we have

$$
\begin{aligned}
(P 1)(x)=\lim _{\delta \rightarrow 0^{+}} \frac{1}{w_{n-1}} \int_{|t-x| \geq \delta} \frac{\overline{t-x}}{|t-x|^{n}} \theta(t) d S t & =0, \\
x & \in \partial \Omega .
\end{aligned}
$$

Moreover, by [15-17] and Lemma 9, we can obtain

$$
\frac{1}{w_{n-1}} \int_{\partial \Omega} \frac{\overline{t-x}}{|t-x|^{n}} \theta(t) d S t= \begin{cases}\frac{1}{2}, & x \in \Omega ; \\ -\frac{1}{2}, & x \in \mathbb{R}^{n} \backslash \Omega .\end{cases}
$$

Since $B \in A_{n}(R)$ and $B(B \neq 0)$ present right inverse element $B^{-1}$, we have $B B^{-1}=B^{-1} B=I$.

Define

$$
Y(x)= \begin{cases}B, & x \in \Omega, \\ 1 & x \in \mathbb{R}^{n} \backslash \Omega .\end{cases}
$$

Obviously, $Y(x)$ is a regular function and there exists an inverse element $Y^{-1}(x)$, and

$$
\begin{aligned}
Y^{+}(x) & =B, \\
Y^{-}(x) & =1, \\
Y^{+}(x)\left[Y^{-1}(x)\right] & =B, \\
D_{x} Y(x) & =0,
\end{aligned}
$$


Therefore, (23) can be transformed into

$$
\begin{aligned}
F^{+}(x)\left[Y^{+}(x)\right]^{-1}= & F^{-}(x)\left[Y^{-}(x)\right]^{-1} \\
& +h(t)\left[Y^{+}(x)\right]^{-1}, \quad x \in \partial \Omega .
\end{aligned}
$$

Denote

$$
H(x)=\frac{1}{w_{n-1}} \int_{\partial \Omega} \frac{\overline{t-x}}{|t-x|^{n}} \theta(t) h(t) B^{-1} d S t ;
$$

it is easily seen that $H(\infty)=0, D_{x} H(x)=0\left(x \in \mathbb{R}^{n} \backslash \Omega\right)$. By applying Lemmas 6 and 10 for $H(x)$ in (34), we obtain

$$
H^{+}(x)-H^{-}(x)=h(x) B^{-1} .
$$

Combining (33) with (35), we have

$$
\begin{aligned}
& F^{+}(x)\left[Y^{+}(x)\right]^{-1}-H^{+}(x) \\
& \quad=F^{-}(x)\left[Y^{-}(x)\right]^{-1}-H^{-}(x), \quad x \in \partial \Omega .
\end{aligned}
$$

It follows from Painleve's theorem (see [18]) that $F(x) Y(x)-$ $H(x)$ is a regular function in $\mathbb{R}^{n}$. Since (23) find a solution in $R_{0}$, thus, by Lemma $11, F(x) Y(x)-H(x)$ is a constant at $x=\infty$; that is, there exists a Clifford constant $C$ such that

$$
F(x) Y(x)-H(x)=C
$$

therefore, we obtain

$$
F(x)=Y(x)(H(x)+C)=Y(x) H(x)+Y(x) C .
$$

From the above discussion, we obtain the main result of this section.

Theorem 14. Under condition (24), BVP (23) has a solution. If a solution $F(x)$ of $(23)$ is a nonzero constant at $\infty$, then, its solution is of the form

$$
\begin{array}{r}
F(x) \\
=\frac{Y(x)}{w_{n-1}} \int_{\partial \Omega} \frac{\overline{t-x}}{|t-x|^{n}} \theta(t) h(t) B^{-1} d S t+Y(x) C, \\
x \in \mathbb{R}^{n} \backslash \Omega,
\end{array}
$$

where $Y(x)$ is determined by (32). ing.

By Theorem 14 and Lemmas 11-13, we obtain the follow-

Theorem 15. Suppose that a solution $F(x)$ of (23) is zero at $\infty$, then (23) find a solution in problem $R_{-1}$, and its solution is given by

$$
F(x)=\frac{Y(x)}{w_{n-1}} \int_{\partial \Omega} \frac{\overline{t-x}}{|t-x|^{n}} \theta(t) h(t) B^{-1} d S t,
$$

\section{SIE in Clifford Analysis}

In Clifford analysis we solve following SIE of the form

$$
\begin{aligned}
K^{0} f & \equiv f(x) P+\frac{1}{w_{n-1}} \int_{\partial \Omega} \frac{\overline{t-x}}{|t-x|^{n}} \theta(t) f(t) d S t \cdot Q \\
& =g(x), \quad x \in \partial \Omega,
\end{aligned}
$$

where $g(t) \in H(\partial \Omega, \beta)(0<\beta<1)$ and $P, Q$ are Clifford constants. $P+Q$ and $P-Q$ have inverse elements and denoted them as $(P+Q)^{-1}$ and $(P-Q)^{-1}$, respectively. $w_{n-1}$ is the same as the before. $\Omega \subset \mathbb{R}^{n}$ is a bounded domain and its boundary $\partial \Omega$ is a smooth, oriented, and compact Lyapunov surface. And $f(t) \in H(\partial \Omega, \beta)(0<\beta<1)$ is an unknown function. When $g(t) \equiv 0$, we call that (41) is a homogeneous equation; otherwise (41) is nonhomogeneous. Since $P+Q$ and $P-Q$ are reversible on $\partial \Omega,(41)$ is called a regular type SIE with Cauchy kernel, and $K^{0}$ is called the corresponding singular operator.

Define a sectionally holomorphic function as follows:

$$
F(x)=\frac{1}{w_{n-1}} \int_{\partial \Omega} \frac{\overline{t-x}}{|t-x|^{n}} \theta(t) f(t) d S t, \quad x \notin \partial \Omega ;
$$

by applying Lemma 6 for (42), we obtain

$$
\begin{array}{r}
f(x)=F^{+}(x)-F^{-}(x), \\
\frac{1}{w_{n-1}} \int_{\partial \Omega} \frac{\overline{t-x}}{|t-x|^{n}} \theta(t) f(t) d S t=F^{+}(x)+F^{-}(x), \\
x \in \partial \Omega .
\end{array}
$$

Putting (43) into (41), one has

$$
F^{+}(x)=F^{-}(x)(P-Q)(P+Q)^{-1}+g(x)(P+Q)^{-1} .
$$

It follows from (42) that $F(\infty)=0$. If (41) has a solution $f(t) \in H(\partial \Omega, \beta)$, then a function $F(x)$ defined by (42) is a solution of (44) in class $R_{-1}$. Conversely, if (44) has a solution $F(x)$ in class $R_{-1}$, then $f(x)$ obtained by the first equality of (43) is a solution of (41). Indeed, by the first equality of (43) and $F(\infty)=0$, we can know that (42) and the second equality of (43) are fulfilled. Substituting (43) into (44), we easily obtain that $f(t)$ satisfies (41). Thus, the problem (41) is equivalent to solving the problem (44) in class $R_{-1}$.

In order to solve (41), we define two functions $Y(x)$ and $H(x)$ as follows:

$$
\begin{aligned}
& Y(x)= \begin{cases}(P-Q)(P+Q)^{-1}, & x \in \Omega \\
1, & x \in \mathbb{R}^{n} \backslash \Omega,\end{cases} \\
& H(x)=\frac{1}{w_{n-1}} \int_{\partial \Omega} \frac{\overline{t-x}}{|t-x|^{n}} \theta(t) g(t)(P+Q)^{-1} d S t, \\
& x \in \mathbb{R}^{n} \backslash \Omega .
\end{aligned}
$$

The remaining discussions are the same as in Section 4. We will not elaborate on that here.

Thus, we have the following conclusions. 
Theorem 16. Suppose that $P+Q, P-Q$ are reversible, then (41) has a solution on $H(\partial \Omega, \beta)$, and its solution is obtained by

$$
f(x)=F^{+}(x)-F^{-}(x),
$$

where

$$
\begin{aligned}
F(x) & =g(x) \tilde{a}+\frac{2}{w_{n-1}} \int_{\partial \Omega} \frac{\overline{t-x}}{|t-x|^{n}} \theta(t) g(t) \tilde{b} d S t \cdot \widetilde{c} \\
\widetilde{a} & =P(P+Q)^{-1}(P-Q)^{-1} \\
\tilde{b} & =(P+Q)^{-1} \\
\tilde{c} & =Q(P-Q)^{-1}
\end{aligned}
$$

Note that, in Theorem 16, if $P=0$, then $Q^{-1}$ must exist. Therefore, we have the following.

Corollary 17. When $P=0,(41)$ is solvable, its solution is still (46), where $F(x)$ in (46) is determined by

$$
F(x)=\frac{2}{w_{n-1}} \int_{\partial \Omega} \frac{\overline{t-x}}{|t-x|^{n}} \theta(t) g(t) Q^{-1} d S t,
$$

$t \in \partial \Omega$.

Proof. It can be proved directly from Theorem 16.

\section{BVP in Quaternion Analysis}

Quaternion analysis researches analytical theory in quaternion algebra $\mathbb{H}$. Quaternion algebra $\mathbb{H}$ is a four-dimensional real vector space with the basis elements $1, i, j, k$, which satisfy the following multiplication rules:

$$
\begin{aligned}
& i^{2}=j^{2}=k^{2}=-1 ; \\
& i j=-j i=k ; \\
& j k=-k j=i ; \\
& k i=-i k=j .
\end{aligned}
$$

Therefore, $\mathbb{H}$ can be written as

$$
\begin{aligned}
\mathbb{H} & =\left\{x\left|x=x_{1}+i x_{2}+j x_{3}+k x_{4}\right| x_{t} \in \mathbb{R}, t\right. \\
& =1,2,3,4\} .
\end{aligned}
$$

Quaternion analysis offers another possibility of generalizing complex function theory to higher dimension, and it has wide applications in mathematical physics, Dirichlet BVPs of higher-order partial differential equations, and other fields. More details about quaternion analysis can be found in the literatures [19-22].

Boundary value problem for quaternion analysis is described as follows: given a bounded domain $\Omega \subset \mathbb{H}$, its boundary $\partial \Omega$ is a smooth, compact, and oriented Liapunov surface. Denote $\Omega^{+}=\Omega, \Omega^{-}=\mathbb{H} \backslash \Omega$. We want to get a function $f(t)$ such that it is regular in $\Omega^{ \pm}$and continuous on
$\Omega^{+}+\partial \Omega$ (or $\Omega^{-}+\partial \Omega$ ), and we have the following boundary value condition:

$$
f^{+}(t)=f^{-}(t) B+h(t), \quad t \in \partial \Omega, B \neq 0,
$$

where $B \in \mathbb{M}$ is a known Clifford constant and $B$ shows the inverse element $B^{-1}$. The known function $h(t)$ is Höldercontinuous on $\partial \Omega$. If $f(\infty)=C$ (constant) with $C \neq 0$, BVP (51) is denoted as $R_{0}$; if $f(\infty)=0$, this case is denoted as $R_{-1}$; if the order of $f(t)$ is $m$ at $\infty$, denote it as $R_{m}$. By Theorems 14 and 16 , we obtain a solution of (51) in $R_{m}$ :

$$
F(q)=\frac{Y(q)}{w_{n-1}} \int_{\partial \Omega} \frac{\overline{t-q}}{|t-q|^{n}} \theta(t) h(t) B^{-1} d S t+\sum_{l=0}^{m} q^{l} a_{l},
$$

where $a_{l} \in \mathbb{H}, q \in \mathbb{M} \backslash \partial \Omega$. $Y(q), w_{n-1}$, and $\theta(t)$ are the same as in Section 4.

\section{Conclusions}

In this paper, we studied some properties of a regular function in Clifford analysis and generalized several classical theorems in $A_{n}(\mathbb{R})$, such as Liouville theorem, Plemelj formula, Cauchy integral theorem, and Cauchy integral formula. By means of these theorems and of the classical boundary value theory, we dealt with the solvability and the explicit solutions for some classes of BVPs and SIEs in Clifford analysis. Here, our method is different from the ones of the classical BVP, and it is novel and effective. Thus, this paper generalizes the theory of the classical BVPs and SIEs. By using our method, other classes of BVPs and SIEs are discussed further in Clifford analysis.

\section{Conflicts of Interest}

The authors declare no conflicts of interest.

\section{Acknowledgments}

This work was supported by the Science and Technology Plan Project of Qufu Normal University (xkj 201606) and the author gratefully acknowledges this support.

\section{References}

[1] N. I. Muskhelishvilli, Singular Integral Equationsï, NauKa, Moscow, Russia, 2002, 2002.

[2] J. K. Lu, Boundary Value Problems for Analytic Functions, World Scientific Publishing, 1993.

[3] F. D. Gakhov, Boundary Value Problems, New York, NY, USA, 1990.

[4] P. Li, "One class of generalized boundary value problem for analytic functions," Boundary Value Problems, vol. 2015, no. 40, 2015.

[5] P. Li and G. Ren, "Some classes of equations of discrete type with harmonic singular operator and convolution," Applied Mathematics and Computation, vol. 284, pp. 185-194, 2016.

[6] H. F. Bueckner, "On a class of singular integral equations," Journal of Mathematical Analysis and Applications, vol. 14, pp. 392-426, 1966. 
[7] Y. Gong, I. T. Leong, and T. Qian, "Two integral operators in Clifford analysis," Journal of Mathematical Analysis and Applications, vol. 354, no. 2, pp. 435-444, 2009.

[8] J. Colliander, M. Keel, G. Staffilani, H. Takaoka, and T. Tao, "Transfer of energy to high frequencies in the cubic defocusing nonlinear Schrodinger equation," Inventiones Mathematicae, vol. 181, no. 1, pp. 39-113, 2010.

[9] G. Ren, U. Kähler, J. Shi, and C. Liu, "Hardy-littlewood inequalities for fractional derivatives of invariant harmonic functions," Complex Analysis and Operator Theory, vol. 6, no. 2, pp. 373-396, 2012.

[10] W. Wang, "On non-homogeneous Cauchy-Fueter equations and Hartogs' phenomenon in several quaternionic variables," Journal of Geometry and Physics, vol. 58, no. 9, pp. 1203-1210, 2008.

[11] G. Gentili, C. Stoppato, and D. C. Struppa, Regular Functions of a Quaternionic Variable, Springer Monographs in Mathematics, Springer, 2013.

[12] R. Abreu-Blaya, J. Bory-Reyes, F. Brackx, H. De Schepper, and F. Sommen, "Cauchy integral formulae in quaternionic hermitean clifford analysis," Complex Analysis and Operator Theory, vol. 6, no. 5, pp. 971-985, 2012.

[13] P. K. Anh, N. M. Tuan, and P. D. Tuan, "The finite Hartley new convolutions and solvability of the integral equations with Toeplitz plus HANkel kernels," Journal of Mathematical Analysis and Applications, vol. 397, no. 2, pp. 537-549, 2013.

[14] F. Catoire and W.-M. Wang, "Bounds on Sobolev norms for the defocusing nonlinear Schrodinger equation on general flat tori," Communications on Pure and Applied Analysis, vol. 9, no. 2, pp. 483-491, 2010.

[15] P. Li, "Two classes of linear equations of discrete convolution type with harmonic singular operators," Complex Variables and Elliptic Equations. An International Journal, vol. 61, no. 1, pp. 6775, 2016.

[16] P. Li, "Generalized convolution-type singular integral equations," Applied Mathematics and Computation, vol. 311, pp. 314323, 2017.

[17] M. A. Darwish, "Monotonic solutions of a convolution functional-integral equation," Applied Mathematics and Computation, vol. 219, no. 22, pp. 10777-10782, 2013.

[18] P. Li, "Some classes of singular integral equations of convolution type in the class of exponentially increasing functions," Journal of Inequalities and Applications, vol. 2017, p. 307, 2017.

[19] N. V. Mau and N. T. Hoa, "On solvability of a boundary value problem for singular integral equations," Southeast Asian Bulletin of Mathematics, vol. 26, no. 2, pp. 235-244, 2002.

[20] Z. Blocki, "Suita conjecture and the Ohsawa-Takegoshi extension theorem," Inventiones Mathematicae, vol. 193, no. 1, pp. 149158, 2013.

[21] P. Li, "Singular integral equations of convolution type with Hilbert kernel and a discrete jump problem," Advances in Difference Equations, vol. 2017, p. 360, 2017.

[22] H. Wang and G. Ren, "Bochner-Martinelli formula for $k$ cauchy-Fueter operator," Journal of Geometry and Physics, vol. 84, pp. 43-54, 2014. 


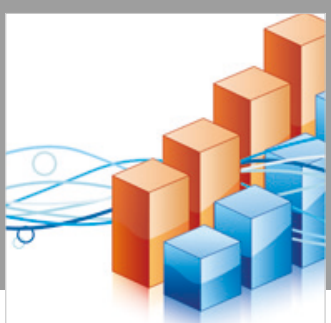

Advances in

Operations Research

\section{-n-m}
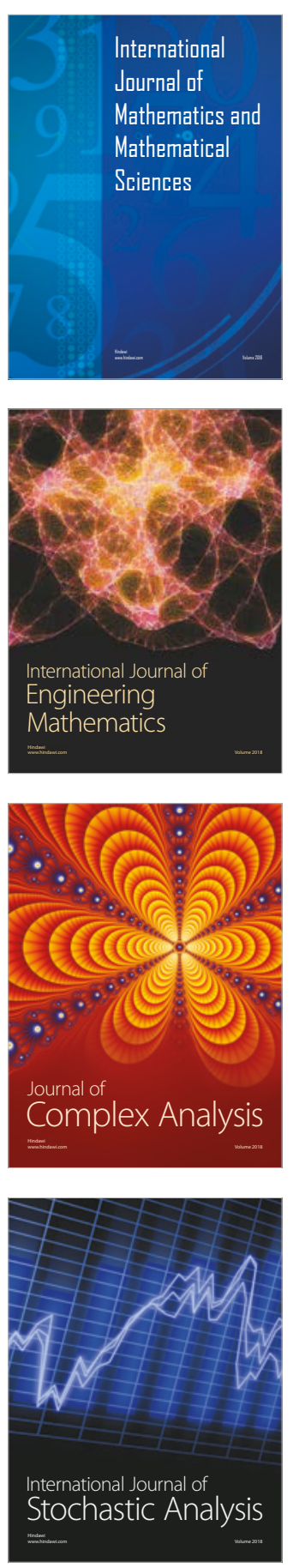
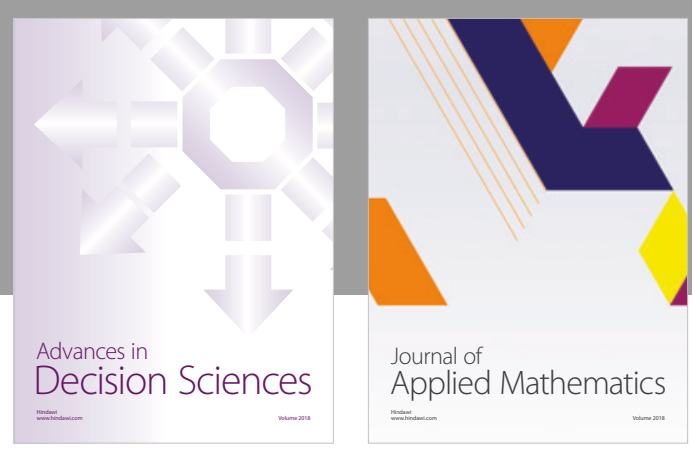

Journal of

Applied Mathematics
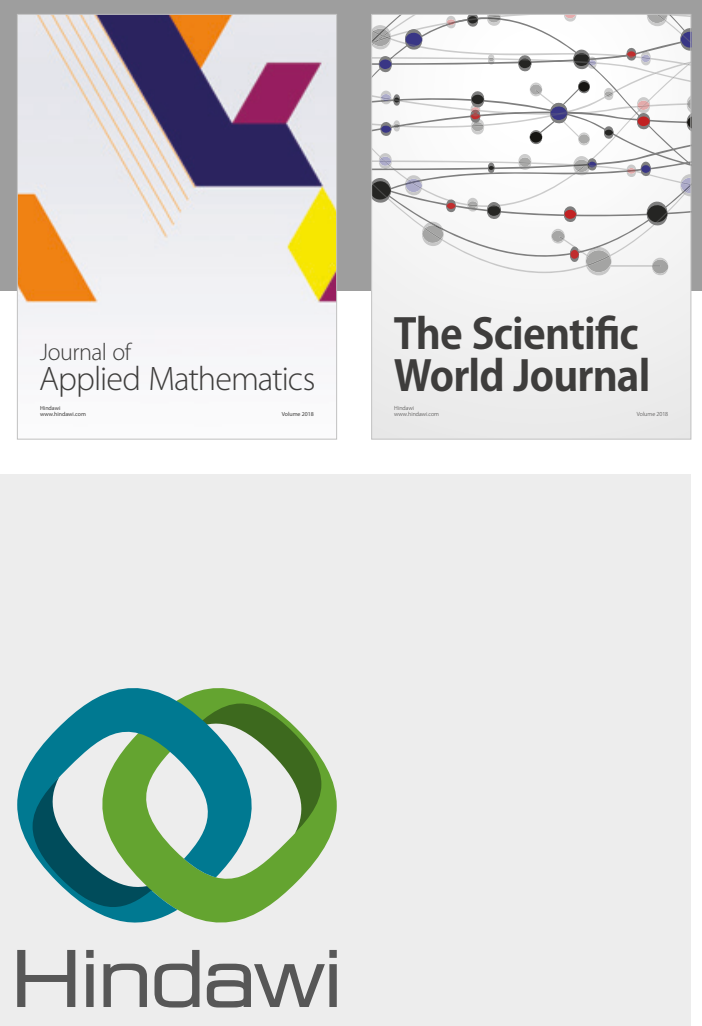

Submit your manuscripts at

www.hindawi.com

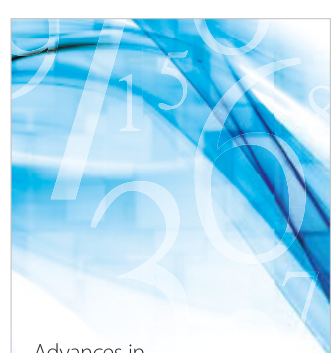

Advances in
Numerical Analysis
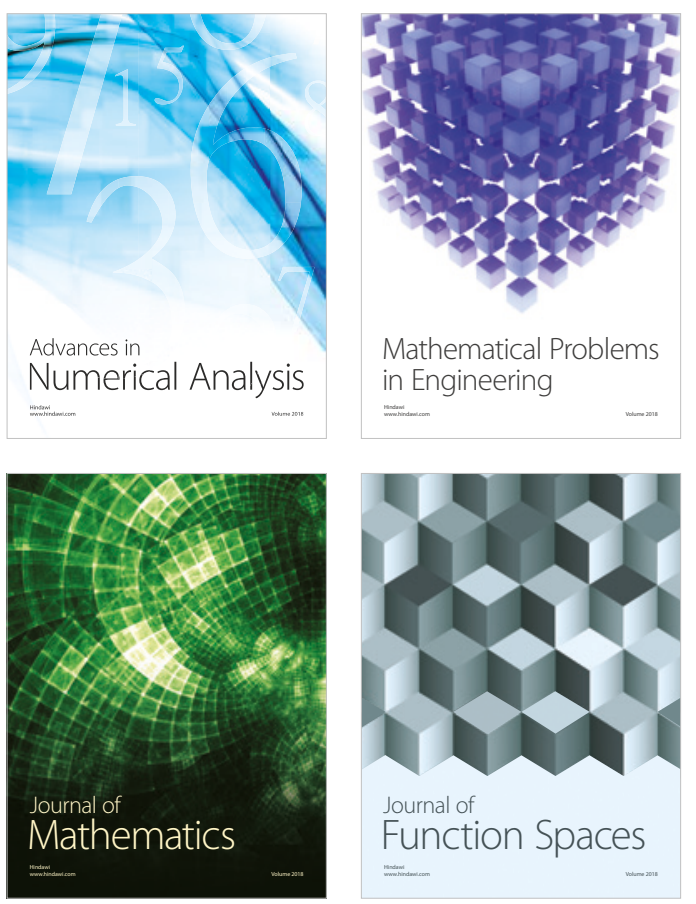

Mathematical Problems in Engineering

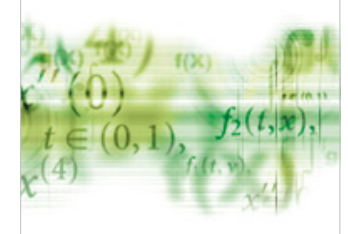

International Journal of

Differential Equations

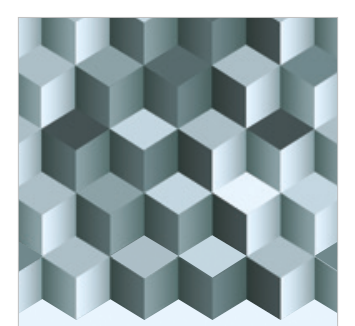

Journal of

Function Spaces

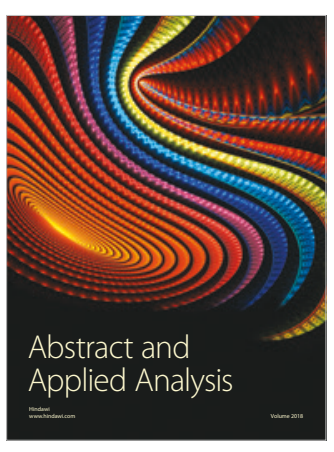

The Scientific

World Journal

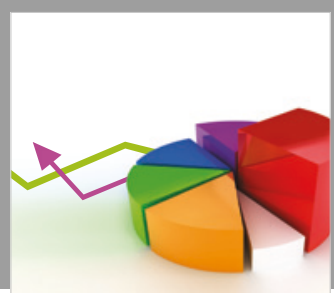

Journal of

Probability and Statistics
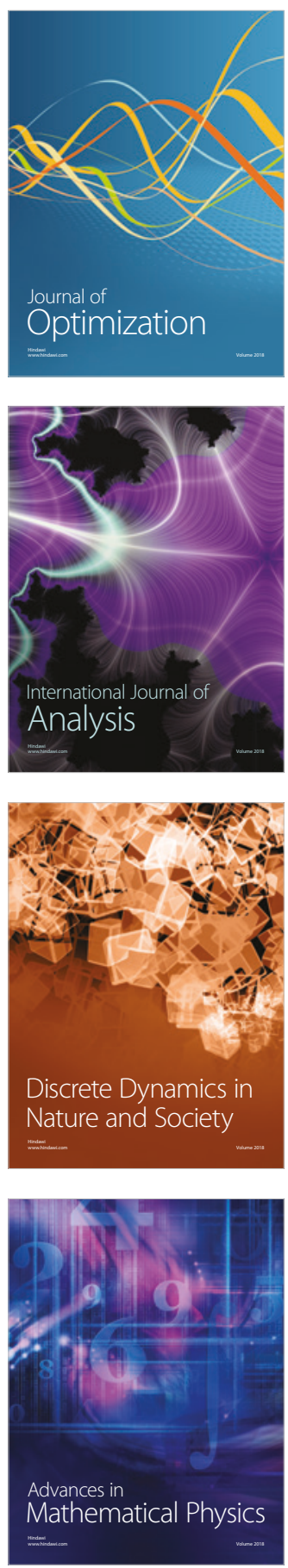